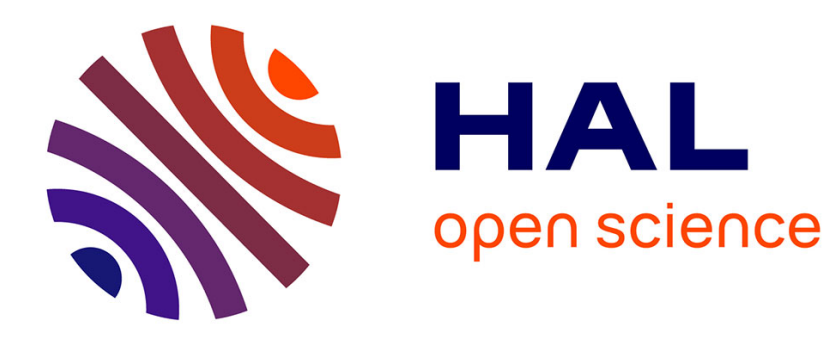

\title{
La réparation économique du préjudice écologique
} N. Dumax

\section{To cite this version:}

N. Dumax. La réparation économique du préjudice écologique. Revue Juridique de l'Environnement, 2009, 1, p. 51 - p. 57 . hal-00617734

\section{HAL Id: hal-00617734 \\ https://hal.science/hal-00617734}

Submitted on 30 Aug 2011

HAL is a multi-disciplinary open access archive for the deposit and dissemination of scientific research documents, whether they are published or not. The documents may come from teaching and research institutions in France or abroad, or from public or private research centers.
L'archive ouverte pluridisciplinaire HAL, est destinée au dépôt et à la diffusion de documents scientifiques de niveau recherche, publiés ou non, émanant des établissements d'enseignement et de recherche français ou étrangers, des laboratoires publics ou privés. 


\section{LA RÉPARATION ÉCONOMIQUE DU PRÉJUDICE ÉCOLOGIQUE}

Nathalie Dumax

Doctorante en économie de l'environnement au laboratoire Gestion des Services Publics à l’École National du Génie de l'Eau et de l'Environnement de Strasbourg (GSP-ENGEES).

Le présent article traite de la partie économique d'un projet interdisciplinaire dans lequel l'ENGEES collabore notamment avec le Centre de Droit de l'Environnement de Strasbourg à l'élaboration d'un outil d'aide à la décision concernant les projets d'aménagement en milieux sensibles. Il s'agit d'un projet mené à l'instigation de Voies Navigables de France (VNF) dont l'application portera sur l'extension des zones portuaires rhénanes françaises qui, compte tenu de la présence au bord du Rhin de zones particulièrement sensibles, pose la question de la prise en compte de l'environnement au sein du processus décisionnel concernant les projets d'aménagement. Après avoir présenté la question de recherche qui se pose aux économistes, nous expliquerons en quoi les méthodes économiques classiques sont insuffisantes puis ce que nous proposons de faire pour y remédier.

\section{I. - QESTION DE RECHERCHE}

Le projet mené par VNF, ainsi que la collaboration interdisciplinaire, ont été mis en place du fait de nouvelles contraintes imposées par la Directive Cadre sur l'Eau (DCE) ${ }^{1}$. En effet, notamment avec son principe de récupération des coûts des services liés aux usages de l'eau, la DCE préconise, en plus des coûts financiers traditionnels, de prendre en compte les coûts pour la ressource, lorsque les usages sont en compétition avec une ressource rare, ainsi que les coûts environnementaux, dommages portés sur l'environnement et les écosystèmes ainsi que sur les personnes qui les utilisent pour leurs activités. Le fait de tenir compte à la fois des coûts économiques, environnementaux et pour la ressource constitue ce que les économistes appellent les coûts complets.

Or, en préconisant l'usage de ces coûts complets, la DCE cherche à intégrer l'ensemble des dommages environnementaux au sein des processus décisionnels à l'origine des politiques publiques mises en œuvre dans le domaine de l'eau. Pour les économistes, cela signifie que le dommage environnemental doit toujours être pris en compte dans son ensemble, notamment au sein d'analyses économiques de type coûts-bénéfices. Le problème qui se pose alors est que pour intégrer complètement les contraintes environnementales, il faut être capable d'évaluer correctement les dommages environnementaux générés par le projet. Or, comme nous le verrons par la suite, lorsque l'on porte atteinte à des milieux naturels complexes cela s'avère particulièrement difficile.

Le terme "environnement" est souvent contesté par certains écologistes et écologues qui y voient notamment une connotation trop anthropocentriste. En effet, les dommages environnementaux ne sont pris en compte qu'à travers l'impact qu'ils peuvent avoir sur les individus et sur leur bien-être. Nous avons néanmoins décidé, en accord avec les juristes, de conserver ce terme. Une distinction sera faite, cependant, entre :

Dommage environnemental dérivé : dommage subi par l'homme du fait de la dégradation de son environnement et conduisant aux préjudices moral et matériel.

\footnotetext{
${ }^{1}$ Directive 2000/60/CE du Parlement européen et du Conseil, du 23 octobre 2000, établissant un cadre pour une politique communautaire dans le domaine de l'eau (JOCE n L 327 du 22 décembre 2000).
} 
Dommage environnemental pur : directement supporté par les milieux naturels en tant que tels et conduisant à ce que l'on appelle « préjudice écologique » ${ }^{2}$.

Cette distinction est nouvelle en économie. Jusqu'à présent, les économistes cherchaient à évaluer le dommage environnemental sans distinction en utilisant des méthodes permettant de mesurer l'impact de ce dommage sur le bien-être des individus, ce qui correspond donc au dommage environnemental dérivé. Cela permettait notamment de déterminer le préjudice moral subi par les individus. Dans ce domaine, les méthodes d'évaluation classiques sont généralement efficaces. Les plus utilisées sont l'évaluation contingente et, plus récemment, l'analyse conjointe.

\section{II. - LIMITES METHODOLOGIQUES}

Outre les limites rencontrées par les méthodes économiques classiques, nous étudierons les limites observées quant à l'application des mesures de compensation telles qu'elle se fait actuellement en France.

\section{A) LIMITES DES MÉTHODES ÉCONOMIQUES CLASSIQUES}

Une fois le fonctionnement des deux principales méthodes utilisées pour l'évaluation des biens et services environnementaux brièvement rappelé, les limites de ces méthodes concernant le cas particulier des systèmes naturels complexes seront soulignées.

\section{Fonctionnement}

L'évaluation contingente est une méthode d'enquête directe qui consiste à aller voir les agents pour leur demander ce qu'on appelle leur « consentement à payer » pour un bien ou un service environnemental ${ }^{3}$. Grâce aux questionnaires administrés par interview direct, par téléphone ou par courrier, une valeur monétaire de l'actif que l'on cherche à estimer peut être obtenue. L'ensemble des données collectées dans le cadre de ces enquêtes est ensuite agrégé de manière à obtenir une valeur monétaire moyenne pour l'actif environnemental en question. Cette méthode fonctionne plutôt bien lorsqu'elle a pour objectif d'évaluer des biens ou des services que les agents connaissent et pour lesquels ils sont capables de donner un prix cohérent. C'est le cas, par exemple, dans les domaines de l'eau et de la santé ${ }^{4}$ qui sont des domaines qui touchent les agents directement et pour lesquels ils ont l'habitude de payer, même si ce n'est pas exactement dans le même contexte.

Les méthodes d'analyse conjointe ${ }^{5}$ sont proches de l'évaluation contingente. Ce sont également des méthodes d'enquête sauf que, dans ce cas, les facteurs environnementaux sont découpés en différentes unités (ou attributs) qui les composent. Il est ensuite demandé aux agents d'évaluer ces unités séparément, par exemple en les classant ou en les notant.

\footnotetext{
${ }^{2}$ V. l'article de P-A. Deetjen, «La traduction juridique du dommage écologique : le préjudice écologique », supra p.39.

${ }_{3}^{3}$ R.C Mitchell et R.T Carson, " Using surveys to value public goods: the contingent valuation method ", Resources for the future, Washington DC, 1989, 463 pages.

${ }^{4}$ A. Rozan, « Une évaluation économique des bénéfices de morbidité induits par une amélioration de la qualité de l’air », Économie et Prévision, 2000, n¹43-144 : p.247-259.

${ }^{5}$ J.-J. Louvière, D.-A. Hensher et J.-D. Swait, « Stated choice methods: analysis and application », Cambridge University Press, 2000, 402 pages.
} 


\section{Limites}

Malheureusement, ces méthodes fonctionnent moins bien lorsqu'il s'agit d'évaluer un dommage environnemental pur. En effet, estimer des systèmes naturels complexes implique de prendre en compte des valeurs dites "d'existence ». Ce sont des valeurs découlant de l'existence même de ces milieux, sans rapport à l'Homme. Concernant l'évaluation contingente, lorsque l'on traite de biens et services peu connus, comme la biodiversité ou les zones naturelles, la méthode semble perdre en fiabilité. En effet, ce sont des systèmes complexes que les agents ne connaissent pas et qu'ils n'arrivent généralement pas à appréhender dans leur ensemble en tenant compte, notamment, de tous les services rendus par ces facteurs environnementaux, ce qui conduit à les sous-estimer ${ }^{6}$.

Au niveau de l'analyse conjointe, la décomposition du système en sous-systèmes, principe même de l'analyse conjointe, ne semble pas optimale pour un système naturel complexe. En effet, même si on parvient à effectuer cette séparation en sous-systèmes, une évaluation distincte de ceux-ci ne tiendrait pas compte des interactions, voire des synergies, existant entre eux, ce qui conduirait, là encore, à les sous-estimer.

Les méthodes d'évaluation classiques ne sont donc pas pertinentes lorsqu'il s'agit de systèmes naturels complexes ${ }^{7}$, ce qui sera forcément le cas pour des projets d'aménagement en milieux sensibles. De nouveaux outils, complémentaires à ceux existants, sont donc nécessaires à l'évaluation du dommage environnemental pur.

\section{B) LIMITES DE LA COMPENSATION}

Dans un tout autre domaine, rappelons que pour le moment, en France, la compensation ${ }^{8}$ se fait au niveau des études d'impact où il est demandé au maître d'ouvrage de supprimer, réduire et, si possible, compenser les dommages environnementaux que son projet devrait engendrer ${ }^{9}$. Néanmoins, si l'on en croit le rapport du Sénat de Laffitte et Saunier $(2007)^{10}$, ce système ne semble pas fonctionner de façon optimale.

D'une part, les projets ayant un impact jugé peu important sur l'environnement ne sont pas pris en compte. Pourtant, pris dans leur ensemble, ces projets pourraient causer des dommages considérables à l'environnement. D'autre part il n'y a, pour le moment, pas de suivi systématique ni de gestion des zones de compensation au-delà du projet. Il n'y a donc aucune garantie concernant la pérennité à moyen et long terme des mesures de compensation effectuées.

\footnotetext{
${ }^{6}$ V. Thèse en Sciences Économiques de D. Laroutis, « Les activités industrielles liées à l'estuaire de la Seine et leurs impacts environnementaux : analyse coûts-bénéfices pour une gestion prospective intégrée », Université de Rouen, Novembre 2006, 313 pages.

${ }^{7}$ P.-A Diamond et J.-A. Hausman, « Contingent valuation : is some number better than no number ? », Journal of Economic Perspectives, 1994, Vol. 8 nº 4 : p. 45-64.

T.H. Stevens, J. Echeverria, R.-J. Glass, T. Hager et T.-A. More, « Measuring the existence value of wildlife: what do CVM estimates really shows? ?, Land Economics, 1991, Vol.67 n4, p.390-400.

${ }^{8}$ V. l'article de M. Lucas, « La compensation environnementale, un mécanisme inefficace à améliorer ? », infra p.59.

${ }^{9}$ Loi no76-629 du 10 juillet 1976 relative à la protection de la nature (JO du 13 juillet).

${ }^{10}$ Rapport du Sénat $n^{\circ} 131$ (2007-2008) de MM. P. Laffitte et C. Saunier, « Les apports de la science et de la technologie au développement durable, Tome II : La biodiversité : l'autre choc ? L'autre chance ? », fait au nom de l'Office parlementaire d'évaluation des choix scientifiques et technologiques, 12 décembre 2007, 192 pages.
} 
D'un autre côté, les législations internationale et européenne tendent de plus en plus à démocratiser l'utilisation des mesures de compensation. En effet, en ce qui concerne le cas particulier des zones humides, la convention Ramsar ${ }^{11}$ envisage dans son article 4.2, des mesures de compensation lorsque « pour des raisons pressantes d'intérêt national » une partie prenante décide de retirer ou de réduire l'étendue d'une zone humide inscrite sur la Liste. Les mesures de compensation apparaissent également dans la législation concernant Natura $2000^{12}$ ainsi que dans la Convention pour la protection du Rhin ${ }^{13}$ (article 4) où une compensation est demandée en cas d'intervention technique majeure.

La compensation constitue donc un système qui, en France, ne fonctionne pas très bien, mais qui tend à se développer à l'international. C'est pourquoi il nous semble intéressant de chercher d'autres moyens permettant d'intégrer ces mesures au sein du processus décisionnel concernant les projets d'aménagement en milieux sensibles.

\section{III.- MÉTHODOLOGIE PROPOSÉE}

La situation telle qu'elle se présente aujourd'hui fait donc état de méthodes économiques limitées d'un côté et d'un principe de compensation qui n'est pas utilisé de façon optimale de l'autre. D'où l'idée proposée d'utiliser le concept de compensation, qui semble se développer, comme un nouveau moyen d'évaluer les dommages engendrés par un projet d'aménagement. L'étude étant conduite dans le cadre d'un projet mené à l'instigation de Voies Navigables de France, l'application de la méthode se fera sur le cas particulier de l'extension des zones portuaires rhénanes françaises. À cet égard, deux concepts développés aux États-Unis dans les années 1980-1990, nous semblent particulièrement intéressants.

\section{A) LE BANKING DE ZONES HUMIDES}

Le banking de zones humides (mitigation banking) a été créé aux États-Unis en 1972 via le Clean Water Act (CWA) pour protéger les zones humides. En effet, selon la section $404 \mathrm{du}$ CWA, le promoteur a l'obligation de compenser tout dommage résiduel causé aux zones humides ${ }^{14}$. Pour ce faire il dispose de quatre moyens d'intervention portant sur des zones humides d'importance équivalente ${ }^{15}$ :

Restaurer : remettre en état un site qui n’est pas le site impacté par le projet mais qui a été endommagé par ailleurs.

Améliorer : intervenir sur un site qui n’a pas été endommagé afin d’y créer de nouvelles fonctions écologiques.

\footnotetext{
${ }^{11}$ La convention relative aux zones humides d'importance internationale particulièrement comme habitats des oiseaux d'eau, Ramsar, le 2 février 1971 (Entrée en vigueur le 21 décembre 1975).

${ }^{12}$ Directive 92/43/CEE du conseil du 21 mai 1992 concernant la conservation des habitats naturels ainsi que de la faune et de la flore sauvages (JOCE $\mathrm{n}^{\circ} \mathrm{L} 206 \mathrm{du} 22$ juillet 1992).

${ }^{13}$ La Convention pour la protection du Rhin du 12 avril 1999, entrée en vigueur le $1^{\text {er }}$ janvier 2003 et ratifiée par la France par la loi n 2002-1004 du 19 juillet 2002 autorisant l'approbation de la convention pour la protection du Rhin (JO du 20 juillet).

${ }^{14}$ C.-C. Holand et M.-E. Kentula, « Impacts of section 404 permits requiring compensatory mitigation on wetlands in California (USA) », Wetland Ecology and Management, 1992, Vol.2 n³ : p.157-169.

${ }^{15}$ A. Breaux, S. Cochrane, J. Evens, M. Martindale, B. Pavlik, L. Suer et D. Benner, « Wetland ecological and compliance assessments in the San Francisco Bay region, California, USA », Journal of Environmental Management, 2005, $\mathrm{n}^{\circ} 74: \mathrm{p} .217-237$.
} 
Créer : utiliser un site où il n’y a pas de milieux naturels pour en créer un nouveau. Cette méthode est rarement utilisée du fait de la difficulté de créer un système naturel complexe sur un site vierge voire pollué et de l’incertitude qui en découle nécessairement.

Protéger juridiquement des zones humides d'importance équivalente. Cette méthode pose également un problème car le projet aboutirait tout de même à une perte nette en milieux naturels.

Dans les cas où l'entrepreneur n'est pas en mesure de mettre en œuvre l'une de ces actions, il a la possibilité de payer une tierce partie pour qu'elle l'effectue à sa place via un marché de compensation $^{16}$. Il s'agit donc d'un mode de compensation a priori : la tierce partie doit effectuer l'une de ces quatre actions avant que le projet ne soit mis en œuvre.

Comment ce système fonctionne-t-il ? Tout d'abord les sites de compensation sont identifiés et les actions mises en œuvre. Ces actions vont donner lieu à des améliorations qui, une fois traduites en crédits équivalents, sont mises à la disposition des banques de compensation où les promoteurs vont pouvoir acheter les unités de fonctions écologiques dont ils ont besoin pour se mettre en conformité avec la loi ${ }^{17}$.

La même procédure est utilisée pour déterminer l'impact qu'un projet d'aménagement va avoir sur l'environnement et les bénéfices apportés par des actions de compensation. C'est l'Habitat Evaluation Procedure, développée dans les années 1980 par l'US Fish and Wildlife Service. Elle permet une équivalence en nature via l'utilisation d'une unité de mesure commune, l'unité d'habitat, constituée du produit d'un index de qualité (compris entre 0 et 1) par la quantité d'habitat considéré ${ }^{18}$.

\section{B) LA METHODE D’ÉQUIVALENCE PAR HABITAT}

La seconde méthode d'évaluation par équivalence en nature est la méthode d'équivalence par habitat (HEA : Habitat Equivalency Analysis). Pratiquée notamment dans le cadre des NRDA (Natural Resource Damage Assessment), il s'agit d'une méthode permettant d'évaluer l'ensemble des coûts à imputer au(x) pollueur(s) dans les cas de pollutions accidentelles (marées noires, déversements de produits toxiques). Elle a été développée par le National Oceanic and Atmospheric Administration (NOAA) dans les années 1990 suite à la parution de l'Oil Pollution Act ${ }^{19}$.

\footnotetext{
${ }^{16}$ V. l'article de M-P. Camproux-Duffrene, « La création d'un marché de biodiversité est-elle possible ? », infra p.69.

${ }_{17}$ W.-A. Weems et L.-W. Canter, « Planning and operational guidelines for mitigation banking for wetland impacts », Environmental Impact Assessment Review, 1995, n¹5, p.197-218.

${ }^{18}$ USFWS, « Habitat as a basis for environmental assessment » U.S. Fish and Wildlife Service, 1980a, 22 pages. USFWS, « Habitat evaluation procedure », US Fish and Wildlife Service, 1980b, 55 pages.

USFWS, "Standard for the development of habitat suitability index models », US Fish and Wildlife Service, 1980c, 53 pages.

${ }^{19}$ V. l'article d'Emilie Cornu-Thenard, « La restauration selon l'US Oil Pollution Act », supra p.27.

NOAA, " Injury assessment - Guidance document for NRDA under the Oil Pollution Act of 1990: damage assessment and restoration program », National Oceanic and Atmospheric Administration, 1996a, 22 pages.

NOAA, « Preassessment phase - Guidance document for NRDA under the Oil Pollution Act of 1990: damage assessment and restoration program », National Oceanic and Atmospheric Administration, 1996b, 190 pages.

NOAA, « Primary restoration - Guidance document for NRDA under the Oil Pollution Act of 1990: damage assessment and restoration program », National Oceanic and Atmospheric Administration, 1996c, 762 pages. NOAA, « Restoration planning - Guidance document for NRDA under the Oil Pollution Act of 1990: damage assessment and restoration program », National Oceanic and Atmospheric Administration, 1996d, 173 pages.
} 
L'objectif de cette procédure consiste non seulement à compenser les dommages environnementaux causés aux écosystèmes endommagés en les restaurant, mais également à compenser les pertes intérimaires ${ }^{20}$ en services écologiques. L'HEA permet de déterminer la taille du projet de restauration compensatoire nécessaire pour compenser entièrement les pertes en services écologiques ${ }^{21}$.

\begin{tabular}{|c|c|c|}
\hline Ressource - Ressource & \multicolumn{2}{|c|}{ Evaluation } \\
\hline $\begin{array}{l}\text { Ressource de même type, qualité } \\
\text { et de valeur comparable }\end{array}$ & \multicolumn{2}{|c|}{$\begin{array}{l}\text { Ressource de même type, qualité } \\
\text { et de valeur non comparable }\end{array}$} \\
\hline \multirow{2}{*}{$\begin{array}{l}\text { Equivalence entre : } \\
\text { Quantité de services naturels } \\
\text { actualisés perdus } \\
\text { et } \\
\text { Quantité de services naturels } \\
\text { actualisés fournis par les actions } \\
\text { de compensation }\end{array}$} & \multicolumn{2}{|c|}{$\begin{array}{l}\text { Equivalence entre: } \\
\text { Valeur des services naturels perdus }\end{array}$} \\
\hline & $\begin{array}{l}\text { Valeur des } \\
\text { services } \\
\text { naturels } \\
\text { fournis par } \\
\text { les actions de } \\
\text { compensation }\end{array}$ & $\begin{array}{l}\text { Coût de } \\
\text { mise en oeuvre } \\
\text { des actions } \\
\text { de compensation }\end{array}$ \\
\hline \multirow[b]{2}{*}{ Equivalence en nature } & Valeur - Valeur & Valeur - Coût \\
\hline & \multicolumn{2}{|c|}{$\begin{array}{l}\text { Méthodes classiques utilisant une } \\
\text { varitété d'unités d'échange }\end{array}$} \\
\hline
\end{tabular}

Fig. 1 : Méthodes d’évaluation utilisées dans le cadre des HEA.

L'HEA utilise deux types de méthode ${ }^{22}$ : l'approche ressource-ressource (également appelée service-service) et l'approche par évaluation. Les deux types de méthode encadrent la question de la taille des mesures de compensation à mettre en œuvre en termes des arbitrages existants entre les services perdus du fait du dommage et les services fournis par les actions potentielles de restauration compensatoire. L'approche ressource-ressource nécessite que les ressources soient de même type, de même qualité et de valeur comparable ${ }^{23}$. Elle détermine la quantité de ressources et de services naturels de remplacement nécessaire pour obtenir une équivalence entre la quantité de services actualisés perdus et la quantité de services actualisés de remplacement fournis par les actions de compensation. C'est donc un échange qui se fait directement en nature. Lorsque les ressources sont de même type, de même qualité mais pas de valeur comparable il est préférable de choisir l'approche par évaluation. Celle-ci détermine

\footnotetext{
NOAA, « Scaling compensatory restoration actions - Guidance document for NRDA under the Oil Pollution Act of 1990: damage assessment and restoration program », National Oceanic and Atmospheric Administration, 1997, 143 pages.

${ }^{20}$ Pertes intérimaires: pertes découlant du fait qu'entre le moment où le dommage à lieu et le retour effectif à l'état initial, les services écologiques ne sont pas fournis.

${ }^{21}$ R.-W. Dunford, T.-C. Ginn et W.-H. Desvousges, « The use of habitat equivalency analysis in natural resource damage assessments », Ecological Economics, 2004, n49, p.49-70.

${ }^{22}$ T. Penn, « A summary of the natural resource damage assessment regulations under the United States Oil Pollution Act », National Oceanic and Atmospheric Administration, 2001.

${ }^{23}$ NOAA, « Habitat Equivalency Analysis : an overview », National Oceanic and Atmospheric Administration, 1995 révisé en 2000 et 2006, 24 pages.
} 
également la taille des mesures de compensation à mettre en œuvre en obtenant une équivalence entre d'un côté la valeur des services perdus et de l'autre soit la valeur des services obtenus grâce aux actions de compensation (approche valeur-valeur), soit le coût de mise en œuvre de ces actions de compensation (approche valeur-coût). Cette méthode utilise les outils d'évaluation économiques classiques mais en posant l'hypothèse que les valeurs peuvent être déterminées par une variété d'unités d'échange incluant les services naturels (notamment grâce à la flexibilité des méthodes d'analyse conjointe). Ces méthodes permettent ainsi d'éviter le recours à une évaluation monétaire via une équivalence en nature.

\section{C) PRÉCISIONS CONCERNANT LA NOUVELLE MÉTHODOLOGIE}

Il ne s'agit pas ici de remplacer les méthodes d'évaluation existantes mais plutôt de les compléter. Ainsi, les méthodes classiques vont permettre de mesurer la variation que le projet engendre sur le bien-être des individus concernés; tandis que la compensation va mesurer directement les coûts que ce même projet fait peser sur l'environnement et sur les services rendus par le milieu atteint non perçus par les agents et donc non pris en compte par les méthodes d'évaluation classiques. Il s'agira alors d'utiliser la méthode d'équivalence employée dans le banking de zones humides et de transposer celle utilisée dans les évaluations de dommages aux États-Unis, afin d'évaluer le dommage environnemental pur ainsi que la taille des actions de compensation qui permettrait de compenser entièrement ce dommage. Le principe de cette nouvelle méthodologie sera ensuite d'utiliser le coût de mise en œuvre de ces mesures de compensation comme correspondant à la valeur des facteurs environnementaux perdus permettant ainsi d'estimer le préjudice écologique subi.

Il est d'ores et déjà possible d'anticiper certaines des difficultés qui ne manqueront pas de se poser lors du développement puis de l'application de cette nouvelle méthode. Pour commencer, des problèmes méthodologiques découleront nécessairement de la transposition d'une méthode généralement utilisée ex post à une application ex ante (c'est le cas de l'Habitat Equivalency Analysis), source d'incertitude. Par ailleurs, ces méthodes d'équivalence nécessitant une quantité importante de données scientifiques, il sera nécessaire de travailler en collaboration avec des scientifiques afin d'identifier, de collecter et de traiter ces données de façon pertinente. Enfin, il sera capital de porter une attention particulière quant à la manière dont ces méthodes complémentaires seront associées de façon à éviter les doubles-comptes. 